\title{
Stringy effects for spinning strings and the Bethe ansatz
}

\author{
Sakura Schäfer-Nameki ${ }^{1,2, *}$ and Marija Zamaklar ${ }^{3, * *}$ \\ ${ }^{1}$ II. Institut für Theoretische Physik der Universität Hamburg, Luruper Chaussee 149, 22761 Hamburg, \\ Germany \\ ${ }^{2}$ Zentrum für Mathematische Physik, Universität Hamburg, Bundesstrasse 55, 20146 Hamburg, Germany \\ ${ }^{3}$ Max-Planck-Institut für Gravitationsphysik, AEI, Am Mühlenberg 1, 14476 Golm, Germany
}

Received 15 December 2005, accepted 15 December 2005

Published online 18 April 2006

Key words AdS/CFT, integrability, Bethe ansatz

PACS 04A25

The one-loop worldsheet corrections to spinning strings in the $s l(2)$ subsector of $A d S_{5} \times S^{5}$ are compared to the quantum string Bethe ansatz. The evaluation of the energy shift is performed in two regimes: a) in the large $\mathcal{J}$ limit, using zeta-function regularization and $b$ ) in the limit of large winding number. The first computation agrees with the Bethe ansatz in the first three orders while the second computation leads to a disagreement with the string Bethe ansatz prediction at leading order. Careful analysis of the zeta-function regularization shows, that in this approach perturbative as well as non-perturbative terms in the string sums are missed. Hence, this together with the result b), implies that the proposed quantum string Bethe equations do not reproduce all terms in the exact string result.

(c) 2006 WILEY-VCH Verlag GmbH \& Co. KGaA, Weinheim

\section{Introduction}

The quantization of strings on $A d S_{5} \times S^{5}$ has to date remained a tantalizing problem. A very promising way to circumnavigate the direct, seemingly impossible quantization of the string is the idea of Arutyunov, Frolov and Staudacher [3] to describe the string spectrum in terms of a set of algebraic equations-the quantum string Bethe equations. The discrete structure of these equations was motivated by the gauge side of the AdS/CFT correspondence, where Bethe ansätze have been established as powerful tools to compute anomalous dimensions of gauge-invariant operators starting from the seminal work of Minahan and Zarembo [4]. The conjectured quantum string Bethe equations were rigorously tested at infinite $\lambda$. However, they could potentially receive $1 / \sqrt{\lambda}$ corrections [3]. It is thus of utmost importance to test these equations against the results from the direct (semiclassical) quantisation of strings at finite values of the coupling.

In the case of string configurations characterised by large quantum numbers, for example spinning string which carry large angular momentum $J$, in addition to the expansion parameter $1 / \sqrt{\lambda}$, an additional small parameter $1 / \mathcal{J}$ appears. In this regime, $1 / \sqrt{\lambda}$ corrections can be traded for $1 / J \sim 1 / \sqrt{\lambda}$ corrections, as long as the new effective coupling $\sqrt{\lambda^{\prime}}=1 / \mathcal{J} \equiv \sqrt{\lambda} / J$ is kept finite. Thus a generic quantity can be organised into a double series in $1 / J$ and $1 / \mathcal{J} \equiv \sqrt{\lambda} / J=\sqrt{\lambda^{\prime}}$. More precisely,

* E-mail: sakura.schafer-nameki@ desy.de

** E-mail: marzam@aei.mpg.de 


$$
\begin{gathered}
E(\mathcal{J}, J)=J\left[1+\frac{1}{\mathcal{J}}\left(c_{0}+\frac{c_{1}}{J}+\frac{c_{2}}{J^{2}}+\ldots\right)+\left(\frac{1}{\mathcal{J}}\right)^{2}\left(\tilde{c}_{0}+\frac{\tilde{c}_{1}}{J}+\frac{\tilde{c}_{2}}{J^{2}}+\ldots\right)\right. \\
\left.+\left(\frac{1}{\mathcal{J}}\right)^{3}\left(\tilde{\tilde{c}}_{0}+\frac{\tilde{\tilde{c}}_{1}}{J}+\frac{\tilde{\tilde{c}}_{2}}{J^{2}}+\ldots\right)+\ldots\right] .
\end{gathered}
$$

Hence, the one-loop (i.e. $1 / \sqrt{\lambda}$ ) worldsheet correction to the energy of spinning strings, in the semiclassical approach maps to "1-loop" $1 / J$ corrections and all "loop" result in the coupling $1 / \mathcal{J}$. Thus computing the the energy shift of the string which is classically confined to the subspace $A d S_{3} \times S^{1}$ and comparing it to the $1 / J$ corrections computed from the quantum string Bethe ansatz [3], provides a direct test of the string Bethe equations. In this article, we review our work on these tests which have appeared in collaboration with K. Zarembo in [1] as well as in [2].

The main conclusion of our work is that the proposed quantum string Bethe equations correctly capture the terms analytic in the coupling $\lambda^{\prime}=1 / \mathcal{J}^{2}$, but they fail to capture terms non-analytic in $\lambda^{\prime}$, as well as non-perturbative corrections, which are present in the string sums. Contrary to the general belief, it turned out $[2,6]$, that unlike in the gauge theory, where the series (1) simplifies, and contains only even powers of $1 / \mathcal{J}$, the string result is of the generic form (1). This is presumably the reason why the quantum string Bethe ansatz [3], being strongly rooted in the discrete structure stemming from the dual gauge theory, fails to capture these terms present in string theory.

\section{Quantum corrections to spinning strings}

In this section we will briefly summarise the main results of [7] on the computation of the semiclassical energy shift for the specific circular spinning string configuration, as well as the explicit evaluation of these results computed in [1]. We consider a circular string spinning in $A d S_{3}$ and rotating with a single spin around a big circle in $S^{5}$ [7]. Global charges of this string (the energy $E$, the $A d S$ spin $S$, and the angular momentum $J$ in the sphere directions) can be combined with the string tension into the following "dimensionless" ratios, which stay finite in the classical $(\lambda \rightarrow \infty, J \rightarrow \infty, S \rightarrow \infty)$ limit

$$
\mathcal{E}=\frac{E}{\sqrt{\lambda}}, \quad \mathcal{S}=\frac{S}{\sqrt{\lambda}}, \quad \mathcal{J}=\frac{J}{\sqrt{\lambda}} .
$$

These quantities are implicitly determined from the equations

$$
\begin{aligned}
\mathcal{E} & =\frac{\kappa \mathcal{S}}{\sqrt{\kappa^{2}+k^{2}}}+\kappa, \\
2 \kappa \mathcal{E}-\kappa^{2} & =2 \sqrt{\kappa^{2}+k^{2}} \mathcal{S}+\mathcal{J}^{2}+m^{2}, \\
k \mathcal{S}+m \mathcal{J} & =0 .
\end{aligned}
$$

As mentioned in the introduction, $1 / \sqrt{\lambda}$ or $1 / J$ can be used interchangeably as the loop counting parameters in the sigma-model. In addition, at any given order in $1 / J$ one can further expand in the BMN coupling $1 / \mathcal{J}^{2}=\lambda / J^{2}$. In this way starting from the energy $\mathcal{E}$, one recovers the two-loop perturbative SYM results.

The one-loop worldsheet correction to this solution was obtained by Park, Tirziu and Tseytlin in [7] and results in the following correction to the classical energy

$$
\delta E^{\text {string }}=\delta E^{(0)}+\delta E^{o s c} .
$$

Here the zero-mode contribution is given by

$$
\delta E^{(0)}=\frac{1}{2 \kappa}\left(4 \nu+2 \kappa+2 \sqrt{\kappa^{2}+\left(1+r_{1}^{2}\right) k^{2}}-8 \sqrt{c^{2}+a^{2}}\right) .
$$


The oscillator part has the following form

$$
\begin{aligned}
\delta E^{o s c}=\frac{1}{\kappa} \sum_{n=1}^{\infty} & \left(4 \sqrt{n^{2}+\nu^{2}}+2 \sqrt{n^{2}+\kappa^{2}}-4 \sqrt{(n+\gamma)^{2}+\alpha^{2}}-4 \sqrt{(n-\gamma)^{2}+\alpha^{2}}\right. \\
& \left.+\frac{1}{2} \sum_{I=1}^{4} \operatorname{sign}\left(C_{I}^{(n)}\right) \omega_{I, n}\right),
\end{aligned}
$$

where the last term is the contribution of the $s l(2)$-modes, which are the four solutions of the quartic equation

$$
\left(\omega^{2}-n^{2}\right)^{2}+4 r_{1}^{2} \kappa^{2} \omega^{2}-4\left(1+r_{1}^{2}\right)\left(\sqrt{\kappa^{2}+k^{2}} \omega-k n\right)^{2}=0 .
$$

The first line corresponds to the transverse and fermionic modes. The various parameters are defined as

$$
\begin{aligned}
\nu & =\sqrt{\mathcal{J}^{2}-m^{2}}, \\
\alpha & =\sqrt{\frac{\kappa^{2}+\nu^{2}}{2}}, \\
r_{1}^{2} & =\frac{\kappa^{2}-2 m^{2}-\nu^{2}}{2 k^{2}}=-\frac{m}{k} \frac{\mathcal{J}}{\sqrt{\kappa^{2}+k^{2}}}, \\
\gamma & =\frac{1}{2} \kappa\left(1+\frac{2 k^{2}\left(1+r_{1}^{2}\right)}{\kappa^{2}-\nu^{2}}\right) \sqrt{\frac{\kappa^{2}-\nu^{2}-2 k^{2} r_{1}^{2}}{2\left(\kappa^{2}+k^{2}\right)}} .
\end{aligned}
$$

The factors $C_{I}^{(n)}$ appearing in (8) are determined from

$$
C_{I}^{(n)}=\left(\omega_{I, n}^{2}-n^{2}\right) \prod_{J \neq I}\left(\omega_{I}-\omega_{J}\right) .
$$

Due to the high complexity of the summands in (6), it seems that these sums cannot be evaluated exactly in analytic fashion. Thus, in order to evaluate the full energy shift, one is forced to expand each summand in $1 / \mathcal{J}$, before performing the summation. Note that each summand admits a regular expansion in $1 / \mathcal{J}^{2}$, i.e. it is analytic in $\lambda^{\prime}$. However, this procedure is not harmless, because the sum is not uniformly convergent and modes with $n \sim \mathcal{J}^{2}$ can give a finite contribution. This is reflected in superficial divergences which arise starting from second order in $1 / \mathcal{J}^{2}$. In [1] we ignored this problem and used zeta-function regularization to sum the divergent series. The resulting coefficients $\delta E_{p}^{\text {string }}$ in

$$
\delta E^{\text {string }}=\sum_{p=1}^{\infty} \frac{\delta E_{p}^{\text {string }}}{\mathcal{J}^{2 p}} .
$$

are then to be compared to the energy shift obtained from the Bethe ansatz. As we shall review in the next section, the result obtained by this "illegal" procedure yields agreement with the quantum string Bethe predictions. However despite this success, careful analysis of zeta-function regularization [2], shows that both zeta function regularisation and string Bethe equations fails to reproduce the same type of terms which are present in the full string sums (6). We shall return to this point in the last section.

\section{The quantum string Bethe ansatz}

\subsection{Classical limit}

Classical solutions for the string moving in $A d S_{3} \times S^{1}$ are uniquely specified by the spectral data of the Lax operator. One can introduce the spectral density $\rho(x)$ defined on a set of intervals $C_{I}=\left(a_{I}, b_{I}\right)$. The 
spectral density satisfies a singular integral equation, which in the $s l(2)$ subsector has the form [5]

$$
2 f d y \frac{\rho(y)}{x-y}=2 \pi k_{I}-2 \pi\left(\frac{\mathcal{J}+m}{x-1}+\frac{\mathcal{J}-m}{x+1}\right), \quad x \in C_{I} .
$$

One refers to this as the classical Bethe equation, since such type of equations arise in the thermodynamic limit of quantum Bethe equations. In addition, the spectral density $\rho(x)$ obeys a set of normalization conditions

$$
\begin{aligned}
& \int d x \frac{\rho(x)}{x}=-2 \pi m, \\
& \int d x \frac{\rho(x)}{x^{2}}=2 \pi(\mathcal{E}-\mathcal{S}-\mathcal{J}), \\
& \int d x \rho(x)=2 \pi(\mathcal{E}+\mathcal{S}-\mathcal{J}) .
\end{aligned}
$$

Here $2 \pi m$ is the total world-sheet momentum which must be quantized because of the periodic boundary conditions on the world-sheet coordinates.

We shall consider the simplest solutions of (13) characterised by only one cut $C=(a, b)$, and hence one mode number $k$. As we will show, this configuration corresponds to the circular string. The presence of only one cut allows us to rewrite the integral equation (13) as an algebraic equation for the resolvant

$$
G(x)=\int d y \frac{\rho(y)}{x-y} .
$$

The normalization conditions for the density (14)-(16) become boundary conditions for $G(x)$

$$
\begin{aligned}
G(0) & =2 \pi m, \\
G^{\prime}(0) & =-2 \pi(\mathcal{E}-\mathcal{S}-\mathcal{J}), \\
\lim _{z \rightarrow \infty} z G(z) & =2 \pi(\mathcal{E}+\mathcal{S}-\mathcal{J}) .
\end{aligned}
$$

Multiplying both sides of (13) by $\rho(x) /(z-x)$ and integrating over $x$ we find the following algebraic equation

$$
G^{2}(z)-2 \pi\left(k-2 \frac{\mathcal{J} z+m}{z^{2}-1}\right) G(z)-2 \pi\left(\frac{\mathcal{J}+m}{z-1} G(1)+\frac{\mathcal{J}-m}{z+1} G(-1)\right)=0 .
$$

The boundary conditions (18)-(20) can be used to eliminate $G( \pm 1)$ from this equation. Expanding (21) at $z=0$ and $z=\infty$ we get

$$
k \mathcal{S}+m \mathcal{J}=0,
$$

and

$$
(\mathcal{J} \pm m) G( \pm 1)=-\pi k(\mathcal{E}+\mathcal{S}-\mathcal{J}) \pm \pi m(k+m) .
$$

The condition (22) imposes rationality on the spins and requires the integers $k$ and $m$ to have opposite signs. We shall assume for definiteness that $m>0$ and $k<0$. Plugging (23) back into (21) we get

$$
G^{2}(z)-2 \pi\left(k-2 \frac{\mathcal{J} z+m}{z^{2}-1}\right) G(z)+\frac{4 \pi^{2}}{z^{2}-1}[k(\mathcal{E}+\mathcal{S}-\mathcal{J}) z-m(k+m)]=0 .
$$


The solution of this quadratic equation is

$$
G(z)=\pi\left(k-2 \frac{\mathcal{J} z+m}{z^{2}-1}\right)+\frac{\pi \sqrt{P(z)}}{z^{2}-1},
$$

where

$$
P(z)=k^{2} z^{4}-4 k(\mathcal{E}+\mathcal{S}) z^{3}+2\left(2 \mathcal{J}^{2}+2 m^{2}-k^{2}\right) z^{2}+4 k(\mathcal{E}-\mathcal{S}) z+k^{2} .
$$

The resolvant determines the density through the discontinuity on the cut

$$
G(x+i 0)-G(x-i 0)=2 \pi i \rho(x), \quad x \in C,
$$

and we find

$$
\rho(x)=\frac{\sqrt{-P(x)}}{x^{2}-1} .
$$

We need one extra condition to express the energy in terms of the spin and the angular momentum. This condition cannot arise from eq. (21). Instead one should look more closely at the structure of the density $\rho(x)$. For general values of the energy, the angular momentum and the spin, the density is real on two cuts, whereas we have assumed that the solution has only one cut. This can be made consistent by requiring that the discriminant of the quartic polynomial (26) is zero, then $P(z)$ has one double root

$$
P(c)=0, \quad P^{\prime}(c)=0 .
$$

These two equations determine the dependence of the energy on the angular momenta, $\mathcal{E}=\mathcal{E}(\mathcal{S}, \mathcal{J})$, in a parametric form and are equivalent to (3), (4) upon the identification

$$
\kappa=-\frac{k}{2}\left(\frac{1}{c}-c\right) .
$$

Hence the resolvent (25) indeed reproduces the classical string configuration (3), (4),(5).

\subsection{Quantum corrections}

If the integral equation (13) is interpreted as the classical limit of some Bethe equations ${ }^{1}$, the density $\rho(x)$ has the meaning of an asymptotic distribution of Bethe roots in the limit when their number (naturally identified with the spin $S$ of the quantum string state) becomes infinite

$$
\rho(x)=\frac{4 \pi}{\sqrt{\lambda}} \sum_{k=1}^{S} \frac{x_{k}^{2}}{x_{k}^{2}-1} \delta\left(x-x_{k}\right) .
$$

The normalization factor $2 \pi / \sqrt{\lambda}$ is the coupling constant of the world-sheet sigma-model. The classical (weak-coupling) limit corresponds to $\lambda \rightarrow \infty$. Because $S$ scales with $\sqrt{\lambda}$ according to (2), the classical limit coincides with the thermodynamic limit, in which the number of roots becomes infinite.

Our starting point are the quantum Bethe equations proposed in $[8,9]^{2}$

$$
\left(\frac{x_{k}^{+}}{x_{k}^{-}}\right)^{J}=\prod_{j \neq k} \frac{x_{k}^{-}-x_{j}^{+}}{x_{k}^{+}-x_{j}^{-}} \frac{1-\frac{1}{x_{k}^{-} x_{j}^{+}}}{1-\frac{1}{x_{k}^{+} x_{j}^{-}}}\left(\frac{1-\frac{1}{x_{k}^{-} x_{j}^{+}}}{1-\frac{1}{x_{k}^{+} x_{j}^{+}}} \frac{1-\frac{1}{x_{k}^{+} x_{j}^{-}}}{1-\frac{1}{x_{k}^{-} x_{j}^{-}}}\right)^{\frac{i \sqrt{\lambda}\left(u_{k}-u_{j}\right)}{2 \pi}},
$$

1 The Bethe ansatz only works for integrable systems, so here we must assume quantum integrability of the world-sheet sigmamodel. There are indeed some indications that integrability is not destroyed by quantum corrections.

2 Although the quantum string can fluctuate in all directions in $A d S_{5} \times S^{5}$, the quantum string Bethe equations have the same number of degrees of freedom as in the pure $s l(2)$ sector. On the gauge theory side different sectors do not talk to each other because operators with different quantum numbers do not mix, but it is not a priori clear why various sectors can be separated on the string theory side. 
where

$$
u_{k}=x_{k}+\frac{1}{x_{k}}
$$

and

$$
x_{k}^{ \pm}+\frac{1}{x_{k}^{ \pm}}=u_{k} \pm \frac{2 \pi i}{\sqrt{\lambda}}
$$

These equations reduce to (13) in the thermodynamic limit when $\sqrt{\lambda}, J, S \rightarrow \infty$. Our goal will be to compute the leading-order quantum correction to the classical Bethe equations.

It might seem that (32) can only give rise to even powers of $1 / \sqrt{\lambda}$, since the equations are invariant under $\sqrt{\lambda} \rightarrow-\sqrt{\lambda}$. Nevertheless the odd powers of $1 / \sqrt{\lambda}$ arise in the expansion and the leading quantum correction is $O(1 / \sqrt{\lambda})$ for the following reason. The Bethe roots $x_{k}$ condense into cuts in the thermodynamic limit such that the distance between nearby roots goes to zero. But the simultaneous limit of $\lambda \rightarrow \infty$ and $x_{k+1}-x_{k} \rightarrow 0$ is singular in the Bethe equations and this singularity gives rise to a local anomaly. The anomaly cancels at the leading order, but contributes to the $1 / \sqrt{\lambda}$ quantum correction. We shall calculate the anomaly directly from the Bethe equations (32). The calculations are rather complicated and the details are given in appendix A in [1]. The resulting equation for the resolvant differs from (24) by a correction term

$$
\begin{gathered}
G^{2}(z)-2 \pi\left(k-2 \frac{\mathcal{J} z+m}{z^{2}-1}\right) G(z)+\frac{4 \pi^{2}}{z^{2}-1}[k(\mathcal{E}+\mathcal{S}-\mathcal{J}) z-m(k+m)] \\
+\frac{4 \pi}{\sqrt{\lambda}} \frac{z^{2}}{z^{2}-1} \int d x \frac{\rho^{\prime}(x) \pi \rho(x) \operatorname{coth} \pi \rho(x)}{z-x}=0 .
\end{gathered}
$$

Solving this quadratic equation we find a density which is of the form (28), where the function $P(z)$ obtains a correction

$$
\delta P(z)=\frac{4 \pi}{\sqrt{\lambda}} \frac{z^{2}\left(1-z^{2}\right)}{\pi^{2}} \int d x \frac{\rho^{\prime}(x) \pi \rho(x) \operatorname{coth} \pi \rho(x)}{z-x} .
$$

The energy can be found as before, from the requirement that there is only one cut present

$$
P(c+\delta c)+\delta P(c+\delta c)=0, \quad P^{\prime}(c+\delta c)+\delta P^{\prime}(c+\delta c)=0 .
$$

Expanding the first equation to linear order we get

$$
\frac{\partial P(c)}{\partial \mathcal{E}} \delta \mathcal{E}+\frac{\partial P(c)}{\partial c} \delta c+\delta P(c)=0 .
$$

Taking into account that $\partial P(c) / \partial c=0$ we find

$$
\delta \mathcal{E}=-\frac{\delta P(c)}{\partial P(c) / \partial \mathcal{E}} .
$$

For $\partial P / \partial \mathcal{E}$ we get from (26)

$$
\frac{\partial P(c)}{\partial \mathcal{E}}=-4 k c\left(c^{2}-1\right) .
$$

Rescaling back to the physical energy we obtain

$$
\delta E^{\text {Bethe }}=\frac{c}{\pi k} \int d x \frac{\rho^{\prime}(x) \pi \rho(x) \operatorname{coth} \pi \rho(x)}{x-c} .
$$


We can also introduce

$$
\tilde{\rho}(x)=\frac{1}{\pi} \int_{0}^{\pi \rho(x)} d \xi \xi \operatorname{coth} \xi
$$

Then integration by parts in (41) yields

$$
\delta E^{\text {Bethe }}=\frac{c}{\pi k} \int d x \frac{\tilde{\rho}(x)}{(x-c)^{2}} .
$$

Comparing the two expressions $\delta E^{\text {Bethe }}$ with the zeta-function regularized expression $\delta E^{\text {string }}$ in an expansion of $1 / \mathcal{J}^{2}=\lambda^{\prime}$ around large $\mathcal{J}$ we have found agreement up to third order.

\section{Limit of large winding number and a mismatch}

To complement the discussion in the last section we now want to do an independent test which avoids the convergence issues mentioned earlier. Namely, we will consider the limit of large winding number $(|k| \gg 1)$, for which the energy shifts can be calculated analytically. In this limit $\mathcal{J}, \mathcal{E}$ and $m$ stay finite, but the spin goes to zero: $\mathcal{S} \ll 1$. The string remains macroscopic in this limit, since it winds around the big circle of $S^{5}$, but its size in $A d S_{5}$ shrinks to zero. We will also have to assume that $\mathcal{J} /|k| \ll 1$, which means that there is no overlap with the perturbative regime we have discussed so far. In fact, the energy shift turns out to depend on $1 / \mathcal{J}=\sqrt{\lambda} / J$ rather than $1 / \mathcal{J}^{2}$ in the large- $k$ limit, and hence it is not possible to compare string quantum corrections to perturbative SYM theory in this regime. The details of the string calculation are given in the original paper [1]. The result is given by

$$
\begin{aligned}
\delta E= & \frac{2 F\left(0, \sqrt{\mathcal{J}^{2}-m^{2}}\right)+2 F(0, \mathcal{J}+m)-4 F\left(\left\{\frac{|k|}{2}\right\}, \sqrt{\mathcal{J}(\mathcal{J}+m)}\right)}{\mathcal{J}+m} \\
& +\sqrt{m \mathcal{J}}+(\mathcal{J}+m) \ln \frac{\sqrt{\mathcal{J}+m}}{\sqrt{\mathcal{J}}+\sqrt{m}}-m,
\end{aligned}
$$

where the function $F(\beta, \alpha)$ is defined as

$$
F(\beta, \alpha) \equiv \sqrt{\alpha^{2}+\beta^{2}}-\beta^{2}+\alpha^{2} \int_{0}^{\infty} \frac{d \xi}{\mathrm{e}^{\xi}-1}\left(\frac{2 J_{1}(\alpha \xi)}{\alpha \xi} \cosh \beta \xi-1\right)
$$

A peculiar property of this result is the dependence on the fractional part of $k / 2$, which means that the large- $k$ limit of the string energy shift depends on whether the winding number $k$ is even or odd. On the other side, this kind of irregularity does not arise in the Bethe ansatz, and also in the zeta-regularized large- $\mathcal{J}$ expansion.

\subsection{Bethe ansatz calculation}

We begin with the classical limit. To take the large- $k$ limit it is convenient to rewrite (26) in the two equivalent forms

$$
P(x)=k^{2}\left(x^{2}-1\right)^{2}-4 k \mathcal{E} x\left(x^{2}-1\right)+4 m \mathcal{J} x(x \pm 1)^{2}+4(\mathcal{J} \mp m)^{2} x^{2} .
$$

The first two terms blow up in the $k \rightarrow \infty$ limit unless $x$ is close to 1 or -1 . The roots of $P, a, b$ and $c$, thus lie in the vicinity of \pm 1 . Changing the variables to

$$
x= \pm 1+\frac{v}{k},
$$


and taking the limit $k \rightarrow \infty$, we get

$$
P(x)=4 v^{2}-8 \mathcal{E} v+4(\mathcal{J} \pm m)^{2}, \quad \text { at } \quad x \rightarrow \pm 1 .
$$

Thus two of the roots of $P(x)$ lie near 1 and two lie near -1 . The double root should lie at $x \approx 1$, from which we find

$$
\mathcal{E}=\mathcal{J}+m
$$

and

$$
c=1-\frac{\mathcal{E}}{|k|}
$$

Solving (48) near $x=-1$, we find the endpoints of the cut

$$
\left\{\begin{array}{l}
b \\
a
\end{array}\right\}=-1-\frac{(\sqrt{\mathcal{J}} \pm \sqrt{m})^{2}}{|k|} .
$$

We see that the cut shrinks to a very small size, whereas the density according to (14)-(16) is still normalized to $O(1)$. Thus the density is highly peaked near -1 . Indeed, from (28) and (48) we find

$$
\rho(x)=\frac{|k|}{v} \sqrt{2(\mathcal{J}+m) v-v^{2}-(\mathcal{J}-m)^{2}} .
$$

The integral (43) can be easily evaluated in the $k \rightarrow \infty$ limit. Because the density is large, $\cosh \xi$ in (42) can be approximated by 1 , and thus

$$
\tilde{\rho}=\frac{\pi}{2} \rho^{2}, \quad \text { at } \quad \rho \rightarrow \infty
$$

We thus get from (43)

$$
\delta E^{\text {Bethe }}=\frac{1}{8 k} \int d x \rho^{2}(x) .
$$

Using $d x=d v /|k|$ and the explicit expression (52) for the density, we find

$$
\delta E^{\text {Bethe }}=\frac{\mathcal{J}+m}{2} \ln \frac{\sqrt{\mathcal{J}}+\sqrt{m}}{\sqrt{\mathcal{J}}-\sqrt{m}}-\sqrt{m \mathcal{J}} .
$$

This clearly disagrees with the string theory calculation (44); in particular the Bethe ansatz result has a regular dependence on $k$.

An independent crosscheck of our observation is provided by performing a numerical evaluation of the sum (6). The same type of deviations between the Bethe ansatz and the string theory computation is also observed numerically both for large and finite values of the parameter $k$. We refer the interested reader to [1] for details.

\section{Remarks on the comparison and issue of zeta function regularisation}

The results of the previous sections clearly point at two conclusions: firstly, the quantum string Bethe ansatz of [3] in the large $\mathcal{J}$ limit captures in a highly non-trivial manner the string result, evaluated using zeta-function regularization. Secondly quantum string Bethe ansatz fails to match the strings prediction 
in the regime of finite $\lambda$ and large $k$, where the computations are done without invoking zeta function regularisation. The important question is how these two results can be simultaneously correct?

As a possible explanation for the incompatibility of these results it was proposed that zeta-function regularization may not correctly sum the semi-classical string result [1]. In [2], by considering several examples of string energy shifts of related nature, which however allow for exact evaluation by other means, we have demonstrated, that zeta function regularization can fail. Furthermore, the explicit analysis of the string sums (in the $s u(2)$ subsector) showed that although the coefficients of $1 / \mathcal{J}^{2 n}$ in the expansion are correctly reproduced by the zeta-function regularisation, the coefficients of $1 / \mathcal{J}^{2 n+1}$ as well as nonvanishing non-perturbative contributions (i.e. of order $e^{-\mathcal{J}}$ ), which are present in the string sum, are not reproduced by zeta function regularisation. Both types of terms are also missed by the quantum string Bethe equations, explaining the origin of the mismatch in the large $k$ regime found in [1].

An important outcome of this analysis is that the terms in the string sums which are not captured by the quantum Bethe equations are non-analytic in the coupling, being proportional to $\left(\sqrt{\lambda^{\prime}}\right)^{2 n+1}$ for integral $n$ and $e^{-1 / \sqrt{\lambda^{\prime}}}$. It remains an important open problem how to modify the S-matrix of [3] in order to incorporate these effects. Some progress in resolving this problem have been made in [6], but much more still remains to be done.

Acknowledgements It is a great pleasure to thank Kostya Zarembo for collaboration on the paper [1] and numerous related discussion. We would also like to thank Niklas Beisert and Arkady Tseytlin for important communications.

\section{References}

[1] S. Schafer-Nameki, M. Zamaklar, and K. Zarembo, J. High Energy Phys. 0509, 051 (2005) [arXiv:hepth/0507189].

[2] S. Schafer-Nameki and M. Zamaklar, J. High Energy Phys. 0510, 044 (2005) [arXiv:hep-th/0509096].

[3] G. Arutyunov, S. Frolov, and M. Staudacher, hep-th/0406256.

[4] J.A. Minahan and K. Zarembo, J. High Energy Phys. 0303, 013 (2003), hep-th/0212208.

[5] V.A. Kazakov and K. Zarembo, J. High Energy Phys. 0410, 060 (2004), hep-th/0410105.

[6] N. Beisert and A.A. Tseytlin, Phys. Lett. B 629, 102 (2005), hep-th/0509084.

[7] I.Y. Park, A. Tirziu, and A.A. Tseytlin, J. High Energy Phys. 0503, 013 (2005); hep-th/0501203.

[8] M. Staudacher, J. High Energy Phys. 0505, 054 (2005) [arXiv:hep-th/0412188].

[9] N. Beisert and M. Staudacher, Nucl. Phys. B 727, 1 (2005) [arXiv:hep-th/0504190]. 